\title{
Stückelberg interferometry using spin-orbit-coupled cold atoms in an optical lattice
}

\author{
Shuang Liang, ${ }^{1}$ Zheng-Chun Li $\odot,{ }^{1}$ Weiping Zhang, ${ }^{2,3}$ Lu Zhou $\odot,{ }^{1,3, *}$ and Zhihao Lan ${ }^{4, \dagger}$ \\ ${ }^{1}$ State Key Laboratory of Precision Spectroscopy, Department of Physics, School of Physics and Electronic Science, \\ East China Normal University, Shanghai 200241, China \\ ${ }^{2}$ Department of Physics and Astronomy, Shanghai Jiaotong University and Tsung-Dao Lee Institute, Shanghai 200240, China \\ ${ }^{3}$ Collaborative Innovation Center of Extreme Optics, Shanxi University, Taiyuan, Shanxi 030006, China \\ ${ }^{4}$ Department of Electronic and Electrical Engineering, University College London, Torrington Place, London WC1E 7JE, United Kingdom
}

(Received 12 June 2020; accepted 1 September 2020; published 23 September 2020)

\begin{abstract}
Time evolution of spin-orbit-coupled cold atoms in an optical lattice is studied, with a two-band energy spectrum having two avoided crossings. A force is applied such that the atoms experience two consecutive Landau-Zener tunnelings while transversing the avoided crossings. Stückelberg interference arises from the phase accumulated during the adiabatic evolution between the two tunnelings. This phase is gauge field dependent and thus provides new opportunities to measure the synthetic gauge field, which is verified via calculation of spin transition probabilities after a double-passage process. Time-dependent and time-averaged spin probabilities are derived, in which resonances are found. We also demonstrate chiral Bloch oscillation and rich spin-momentum locking behavior in this system.
\end{abstract}

DOI: 10.1103/PhysRevA.102.033332

\section{INTRODUCTION}

Atom interferometry has been proven to be a powerful tool for precision metrology [1]. In addition, atom interferometry can be used to test fundamental physical theory such as general relativity [2-6]. Recent years have also witnessed growing interest in atom interferometry with synthetic gauge fields [7-10], mostly due to the fact that synthetic gauge fields such as spin-orbit (SO) coupling can be generated in neutral cold atoms via atom-light interaction [11-13]. The gauge field can couple the atom's internal spin states to its center-of-mass motion, thus the path difference in coordinate space can be mapped to a spin interference signal in an interferometer setup, which can be used to measure external $\mathrm{AC}$ force [7] and demonstrate the non-Abelian Aharonov-Bohm effect $[9,10]$. It is interesting to note that non-Abelian gauge fields in real space have been observed recently [14] and a five-dimensional non-Abelian gauge field has also been explored $[15,16]$.

In this work we concentrate on Stückelberg interferometry. The principle of Stückelberg interference lies in a quantum system traveling through more than one avoided crossing of two of its energy levels [17-20]. Avoided crossing represents a close encounter of two energy levels without actual degeneracy [21]. Passing through the avoided crossings leads to Landau-Zener (LZ) tunneling, which coherently splits and recombines wave function between the two energy levels. Thus the phase accumulated between two LZ transitions (sometimes referred as the Stückelberg phase) leads to quantum interference. The avoided crossings function as beam splitters in parameter (quasimomentum or time) space, in the sense

\footnotetext{
*1zhou@phy.ecnu.edu.cn

†z.lan@ucl.ac.uk
}

that the physical principle underlying Stückelberg interferometry is identical to that of the Mach-Zehnder interferometer. Stückelberg interferometry has been implemented in molecule formation in ultracold atoms [22], atomic Bose-Einstein condensates (BECs) in an optical lattice [23,24], electronic spins in a nitrogen-vacancy center [25], and, recently, acoustic modes [26]. The interband LZ tunneling of ultracold atoms in bichromatic optical lattices [27-29] and between high-energy bands [30-32] is also predicted to have the potential to perform Stückelberg interference.

Recently a periodically driven SO-coupled atomic BEC in free space was used to implement Stückelberg interference [33]. Using periodic modulation of Raman coupling to create a pair of avoided crossings in the energy dispersion, the resulting interference fringes become modulation frequency dependent. Besides scenarios in free space, synthetic SO coupling has also been implemented in ultracold atomic gas trapped in an optical lattice via either optical clock transition [34-36], a double-well optical lattice [37,38], Raman dressing $[39,40]$, or use of a two-dimensional manifold of momentum states [41]. By considering spin as a synthetic dimension, these experiments can resemble the model of a two-leg ladder subject to a magnetic flux [42]. A two-leg ladder has been realized using a single optical cavity with two independent synthetic dimensions [43] and the tunneling problem of a ladder system has also been addressed with an electronic setup [44,45]. Many interesting phenomena such as chiral Bloch oscillation, bandgap closing, edge states, and unconventional phases have been predicted in such a system by incorporating diagonal couplings, additional legs, and strong and long-range interactions [46-62] .

Motivated by these developments, in this work we consider Stückelberg interferometry using SO-coupled cold atoms in an optical lattice. We found the conditions to realize two 
avoided crossings in the energy dispersion of this two-band system, which can be achieved in experiment, and thus the system can be used to perform Stückelberg interference. Stückelberg interferometry represents atom interferometry with synthetic gauge fields and provides a new opportunity to study novel SO-coupled band structures. We demonstrate that the interference pattern reveals a phase which depends on the synthetic magnetic flux, from which information on the synthetic gauge field can be derived. Although the synthetic magnetic flux can be directly probed via measurement of the atomic SO momentum transfer, here Stückelberg interference provides an alternative way to demonstrate the effect of the synthetic gauge field without inquiring about the atomic momentum information. The time evolution is also explicitly studied, where resonances and chiral Bloch oscillation are predicted.

The article is organized as follows: In Sec. II we present our model and the effective Hamiltonian is derived. The principle of Stückelberg interference is illustrated in Sec. III. We use the adiabatic-impulse model to analyze a double-passage process in which two avoided crossings are transversed. A more general discussion of the dynamics is also performed with Floquet-Bloch theory. Section IV is devoted to the discussion of chiral Bloch oscillation in the case where no LZ transitions take place, and finally, we conclude in Sec. V.

\section{MODEL}

In this work we consider the following model, typically representing a two-leg bosonic ladder pierced by a magnetic flux, which can be described by the Hamiltonian $(\hbar=1)$

$$
\begin{aligned}
\hat{H}= & -\frac{\Delta}{2} \sum_{l}\left(\hat{c}_{l}^{\dagger} e^{i \phi \hat{\sigma}_{z}} \hat{c}_{l+1}+\text { H.c. }\right) \\
& +\sum_{l} \hat{c}_{l}^{\dagger}\left(\frac{\Omega}{2} \hat{\sigma}_{x}-\frac{\delta}{2} \hat{\sigma}_{z}\right) \hat{c}_{l} .
\end{aligned}
$$

Hamiltonian (1) can be implemented in the system of Ramandressed ${ }^{87} \mathrm{Rb}$ cold atoms trapped in a one-dimensional optical lattice $[12,39,71]$ along the $z$ direction under the lowest energy band truncation and tight-binding approximation, as shown in Fig. 1(a). Here the atomic hyperfine states $|1,-1\rangle(|\downarrow\rangle)$ and $|1,0\rangle(|\uparrow\rangle)$ are coupled via Raman lasers, thus generating effective SO interaction. By considering the atomic pseudospin as a synthetic dimension, the system can be exactly mapped to a two-leg bosonic ladder, as shown in Fig. 1(b). The spin-momentum locking in the Raman transition is equivalent to spin-dependent (leg-dependent) tunneling between neighboring sites, thus a particle hopping around an elementary plaquette picks up an Aharonov-Bohm phase $2 \phi$, which is equivalent to the presence of an effective magnetic flux $2 \phi$ per plaquette piercing the system. $\phi=\pi k_{R} / k_{l}$, with $k_{R(l)}$ the wave vector of Raman beams projected onto the $z$ axis and the laser forming the lattice, respectively. Here the two-component annihilation operator $\hat{c}_{l}=\left(\hat{c}_{l \uparrow}, \hat{c}_{l \downarrow}\right)^{T}$ and $\Delta$ is half-bandwidth on the scale of $\mathrm{kHz}$ in typical experiments with ${ }^{87} \mathrm{Rb}$ atoms [63]. The interleg coupling is characterized by the hopping amplitude $\Omega / 2$ and detuning $\delta$.

Compared with the previously studied two-leg ladder [42], here the interleg detuning $\delta$ is additionally taken into account.
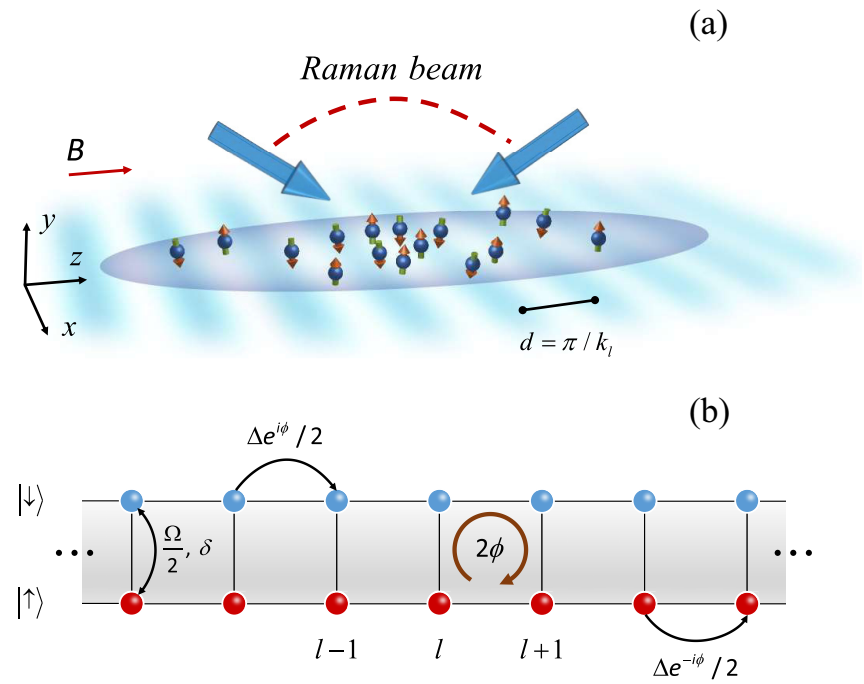

FIG. 1. Schematic showing the system under consideration. (a) Setup: ${ }^{87} \mathrm{Rb}$ cold atoms are confined in a one-dimensional optical lattice along the $z$ direction, inside which the effective SO interaction is induced via coupling of the $|\downarrow\rangle$ and $|\uparrow\rangle$ hyperfine states with Raman lasers. A bias magnetic field $B$ causing quadratic Zeeman splitting is applied along the $z$ direction. (b) The hyperfine states can be treated as an effective synthetic dimension made by two sites connected with a coherent tunneling, resulting in a two-leg ladder pierced by a synthetic magnetic flux $2 \phi$ per plaquette.

We note that the two-photon detuning $\delta$ is usually available via the bias magnetic field in typical experiments with Ramandressed BECs $[11,37,39]$. Making the Fourier transform $\hat{c}_{q}=$ $\sqrt{\frac{d}{2 \pi}} \sum_{l} \hat{c}_{l} e^{-i q l d}$ with $\hat{c}_{q}=\left(\hat{c}_{q \uparrow}, \hat{c}_{q \downarrow}\right)^{T}$ and $d=\pi / k_{l}$ the lattice constant, which is equivalent to the transform of the system from the Wannier basis to the Bloch basis, Hamiltonian (1) can be rewritten in the quasimomentum basis as $\hat{H}=\sum_{q} \hat{c} \hat{\mathcal{H}}_{q} \hat{c}_{q}$, with

$$
\begin{aligned}
\hat{\mathcal{H}}_{q}= & -\Delta \cos \phi \cos (q d) \hat{\mathbb{1}}+\frac{\Omega}{2} \hat{\sigma}_{x} \\
& +\left[-\frac{\delta}{2}+\Delta \sin \phi \sin (q d)\right] \hat{\sigma}_{z} .
\end{aligned}
$$

Hamiltonian $\hat{\mathcal{H}}_{q} \quad$ indicates a two-band structure with $\quad \varepsilon_{ \pm}(q)=\quad-\Delta \cos \phi \cos (q d) \pm$ $\sqrt{[-\delta / 2+\Delta \sin \phi \sin (q d)]^{2}+(\Omega / 2)^{2}}$. When $|\delta / 2 \Delta \sin \phi|$ $<1$, there are two avoided crossings (at which spacing between $\varepsilon_{ \pm}$takes the minimal value $\Omega$ ) located in the first Brillouin zone, thus making the system ideal for implementing Landau-Zener-Stückelberg interferometry.

\section{STÜCKELBERG INTERFEROMETRY}

The principle of Stückelberg interferometry is illustrated in Fig. 2, where we assume $\delta / 2 \Delta \sin \phi>0$ without loss of generality. The two avoided crossings are then located at $A$ with $q_{A} d=\arcsin (\delta / 2 \Delta \sin \phi)$ and $B$ with $q_{B} d=\pi-\arcsin (\delta / 2 \Delta \sin \phi)$. Suppose that the system is initially prepared in the state $\mid q_{0}$, $\left.\uparrow\right\rangle$ with $q_{0} d \in$ 


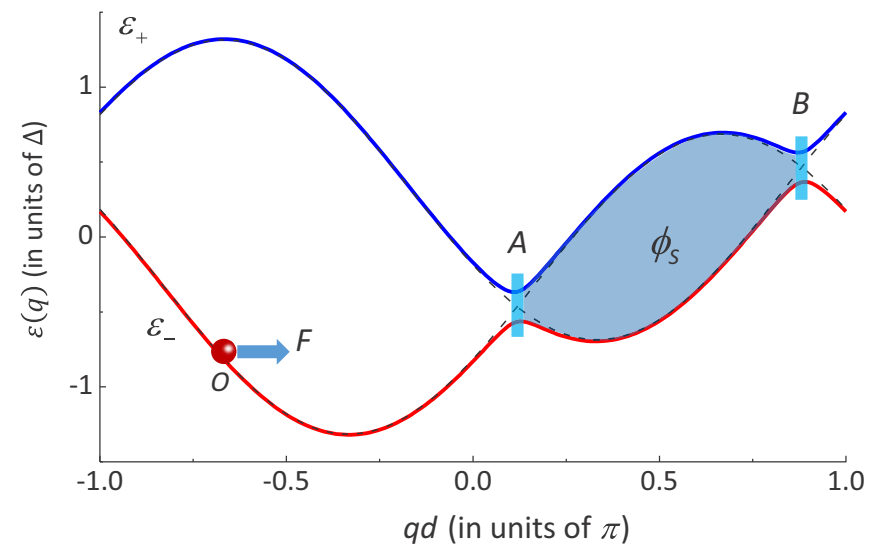

FIG. 2. Energy band $\varepsilon(q)$ at $\delta=0.63 \Delta, \Omega=0.2 \Delta$, and $\phi=$ $\pi / 3$. The diabatic energy levels are shown by dashed lines. This figure illustrates the principle of the two-leg ladder as Stuickelberg interferometry.

$[-\pi, \arcsin (\delta / 2 \Delta \sin \phi)]$ (for example, $q_{0}$ could be the quasimomentum of the system ground state), which is marked as point $O$ in Fig. 2. Furthermore, a constant external force $F$ is exerted on the atoms via tilting of the optical lattice, which drives Bloch oscillation of the atoms. Bloch oscillation depicts the traveling of atoms along the energy bands. Upon reaching the avoided crossing $A$, the atoms will coherently split into two components via LZ transition. The two components then separately travel along $\varepsilon_{ \pm}(q)$ and thus acquire a different phase. Finally, the two components recombine and interfere with each other after another LZ transition at the avoided crossing $B$, from which the information on the phase difference $\phi_{S}$ accumulated during traversing $\varepsilon_{ \pm}(q)$ between $A$ and $B$ can be derived.

In the following we first study this double-passage process (the avoided crossing region is passed twice) using the adiabatic-impulse model in Sec. III A, where a simple relation between the spin population and the Stückelberg phase is found. This model can help us gain physical insight into Stückelberg interferometry. Then the spin dynamics is studied in Sec. III B, from which one can calculate the time-averaged spin population. This is relevant to experiments and the corresponding interference patterns are identified.

\section{A. Double-passage: Adiabatic-impulse model}

Under the assumption that $F$ is weak enough not to induce interband transitions, the adiabatic approximation can be applied, under which the atoms move adiabatically along the energy band with the quasimomentum $q(t)=q_{0}+F t$ [64] except in the vicinity of the avoided crossings. The nonadiabatic evolution of atoms while traversing the avoided crossing region is considered to take place instantaneously, which validates at $\Omega^{2}+(2 \Delta \sin \phi)^{2} \gg(F d)^{2}$ [65]. The double-passage process from $t_{i}=t_{A}^{-}$to $t_{f}=t_{B}^{+}$with $t_{A(B)}=\left(q_{A(B)}-q_{0}\right) / F$ can be described by a transfer matrix $\mathcal{T}_{D}$ in the diabatic basis (bare spin basis) as

$$
c\left(t_{f}\right)=\mathcal{T}_{D} c\left(t_{i}\right)
$$

where $c(t)=\left\langle\hat{c}_{q}\right\rangle=\left(c_{\uparrow}, c_{\downarrow}\right)^{T}$ represents the atomic population amplitude in the diabatic basis (bare spin basis) and is governed by the equations of motion $i d c(t) / d t=\hat{\mathcal{H}}_{q} c(t)$. In the adiabatic-impulse approximation [66], $\mathcal{T}_{D}$ can be divided into three parts.

$i$. The LZ transition at the avoided crossing A: To symmetrize the two diabatic energy levels, we treat $c(t) \exp \left\{i \Delta \cos \phi \int_{0}^{t} d t^{\prime} \cos \left[q\left(t^{\prime}\right) d\right]\right\}$ as the wave vector, submit it to the equations of motion, and get

$$
i \frac{d c_{\uparrow(\downarrow)}}{d t}=\frac{\Omega}{2} c_{\downarrow(\uparrow)} \pm\left[-\frac{\delta}{2}+\Delta \sin \phi \sin (q d)\right] c_{\uparrow(\downarrow)} .
$$

In the vicinity of $A$ with $\left|F d\left(t-t_{A}\right)\right| \ll 1$, Eq. (4) can be linearized as

$$
i \frac{d c_{\uparrow(\downarrow)}}{d t^{\prime}}=\frac{\Omega}{2} c_{\downarrow(\uparrow)} \pm \frac{v}{2} t^{\prime} c_{\uparrow(\downarrow)}
$$

with $\quad v=2 \Delta F d \sin \phi \cos \left(q_{A} d\right)=2 \Delta F d \sin \phi$ $\sqrt{1-(\delta / 2 \Delta \sin \phi)^{2}}$ and $t^{\prime}=t-t_{A}$. Equation (5) defines the standard LZ problem for which the exact solution can be expressed in terms of parabolic cylinder functions $[17,19]$. Then the nonadiabatic transition is described by $\left[c_{\uparrow}(+0), c_{\downarrow}(+0)\right]^{T}=\mathcal{T}_{A}\left[c_{\uparrow}(-0), c_{\downarrow}(-0)\right]^{T}$ with the time-independent matrix

$$
\mathcal{T}_{A}=\left(\begin{array}{cc}
\sqrt{P_{\mathrm{LZ}}} & \sqrt{1-P_{\mathrm{LZ}}} e^{-i \varphi_{\mathrm{st}}} \\
-\sqrt{1-P_{\mathrm{LZ}}} e^{i \varphi_{\mathrm{st}}} & \sqrt{P_{\mathrm{LZ}}}
\end{array}\right),
$$

where the LZ transition probability $P_{\mathrm{LZ}}=\exp (-2 \pi \xi)$ and the Stokes phase $\varphi_{\text {st }}=\pi / 4+\xi(\ln \xi-1)+\arg \Gamma(1-i \xi)$, with $\xi=\Omega^{2} / 4 v$ and the gamma function $\Gamma$.

ii. The adiabatic evolution in the region between the two avoided crossings $A$ and B: One can note that in this region far from the avoided crossings the diabatic energy for spin- $\uparrow$ (spin- $\downarrow$ ) components coincides with the energy dispersion $\varepsilon_{ \pm}(q)$, respectively, i.e., the excited eigenstate of $\hat{\mathcal{H}}_{q}$ coincides with spin- $\uparrow$ while the ground eigenstate coincides with spin- $\downarrow$, as shown in Fig. 2. This enables us to write the evolution matrix as

$$
\mathcal{U}=\left(\begin{array}{cc}
e^{-i \frac{\phi_{S}}{2}} & 0 \\
0 & e^{i \frac{\phi_{S}}{2}}
\end{array}\right)
$$

with $\phi_{S}=\int_{q_{A}}^{q_{B}} d q\left[\varepsilon_{+}(q)-\varepsilon_{-}(q)\right] / F$.

iii. The LZtransition at the avoided crossing $B$ : This process is identical to part i except that $v \rightarrow-v$ in Eq. (5). Then the LZ transition matrix $\mathcal{T}_{B}=\mathcal{T}_{A}^{T}$. form

Combining processes $\mathrm{i}-\mathrm{iii}$, the transfer matrix $\mathcal{T}_{D}$ has the

$$
\begin{aligned}
\mathcal{T}_{D} & =\mathcal{T}_{B} \mathcal{U} \mathcal{T}_{A}=\left(\begin{array}{cc}
\alpha & \beta \\
\beta & \alpha^{*}
\end{array}\right), \\
\alpha & =P_{\mathrm{LZ}} e^{-i \frac{\phi_{S}}{2}}+\left(1-P_{\mathrm{LZ}}\right) e^{i\left(\frac{\phi_{S}}{2}+\varphi_{\mathrm{st}}\right)}, \\
\beta & =-2 i \sqrt{P_{\mathrm{LZ}}\left(1-P_{\mathrm{LZ}}\right)} \sin \left(\frac{\phi_{S}}{2}+\varphi_{\mathrm{st}}\right) .
\end{aligned}
$$

Equations (3) and (8) indicate that the transition probability from spin- $\uparrow$ to spin- $\downarrow$ is $|\beta|^{2}$, which oscillates with the phase $\phi_{S} / 2+\varphi_{\text {st }}$. Since $\phi_{S} \propto 1 / F$, then under the adiabatic approxiation with small force $F$ the Stückelberg phase $\phi_{S}$ is dominant and the contribution from $\varphi_{\text {st }}$ can be neglected [44], i.e., one 

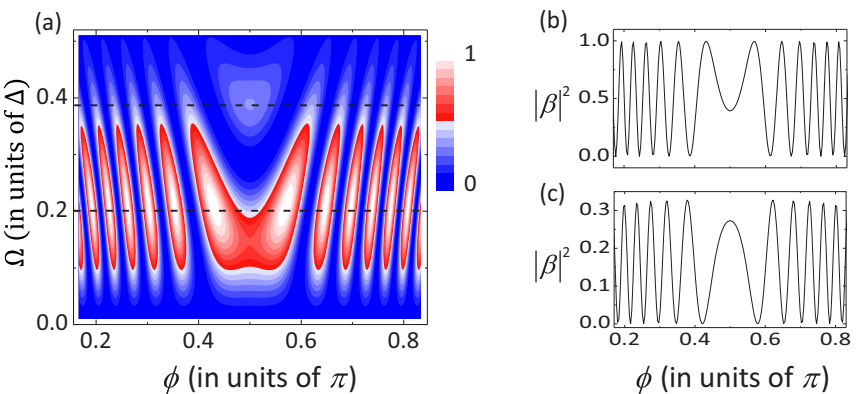

FIG. 3. (a) Contour plot of the transition probability $|\beta|^{2}$ in the $(\phi, \Omega)$ parameter plane with $\delta=0.23 \Delta$ and $F d=0.05 \Delta$. The dashed lines represent $\Omega=0.2 \Delta$ and $0.39 \Delta$, respectively, with the corresponding oscillations shown in (b) and (c).

can neglect the quantum phase and focus on the semiclassical lattice effect.

We calculate the transition probability $|\beta|^{2}$ as a function of the synthetic magnetic flux $\phi$ and the Raman coupling strength $\Omega$ with the results shown in Fig. 3. This figure is symmetric with respect to $\phi=\pi / 2$, as $|\beta|^{2}$ is a function of $\sin \phi$. On both sides of $\phi=\pi / 2$ it displays approximately periodic oscillation which can be recognized as Stückelberg oscillation. The appearance of Stückelberg oscillation versus $\phi$ reflects the nearly monotonic dependence of the Stückelberg phase $\phi_{S}$ on $\phi$ since the value of $P_{\mathrm{LZ}}$ is insensitive to the variation of $\phi$. The interval between two neighboring maximal (minimal) transition probabilities is then given by $\delta \phi_{S} \approx 2 \pi$. The calculation is performed with $F d=0.05 \Delta$ and the oscillation will become stronger for a smaller force $F$. For the parameters considered here, at $\Omega=0.2 \Delta$ we have $P_{\mathrm{LZ}} \approx 0.5$ and hence the transition probability can reach the maximum 1, as shown in Fig. 3(b). Since $\Omega$ determines the energy spacing at the avoided crossings and the LZ transition probability $P_{\mathrm{LZ}}$ is exponentially dependent on it, one can then generally expect that with increasing deviation from $\Omega=0.2 \Delta$ the transition probability $|\beta|^{2}$ will gradually decrease. This is the case except for the region around $\phi=\pi / 2$. As $\phi_{S}$ is a complex function of $\Omega$, another local maximum of the transition probability appears around $\Omega=0.39 \Delta$, as shown in Fig. 3(c).

By measuring the Stückelberg interference fringes one can map out the novel band structure with SO coupling [23,33]. Compared with the experiment [33], here the Raman coupling is not periodically modulated. However, in the scenario with an optical lattice, it can have the effect of engineering the dispersion and realizing two avoided crossings, thus making Stückelberg interference feasible. The interference fringes in [33] rely on the frequency and amplitude of the periodical modulation applied on the Raman beams, showing the effect of Floquet engineering on SO-coupled bands. Here the physics underlying Stuickelberg interference is the presence of synthetic magnetic flux. In the meanwhile, considering the fact that the center of interference (or zeroth-order fringe corresponding to $\phi=\pi / 2$ ) can be easily identified due to the symmetric nature of the interference pattern shown in Fig. 3, it enables one to recognize the order of interference fringe which can be used to indicate the synthetic magnetic flux $\phi$. In this sense the Stückelberg interferometer demonstrated here also provides a new opportunity to measure the synthetic gauge field.

\section{B. Dynamics: Floquet-Bloch theory}

The dynamics considered here is mathematically similar to Bloch oscillation in a two-band system with interband coupling, however, many previous works have dealt with this kind of problem $[67,68]$ without SO coupling. Here we perform the analysis in terms of the Floquet-Bloch operator $[32,69]$. We can start from Hamiltonian (1) with an additional term $-F d \sum_{l} l \hat{c}_{l}^{\dagger} \hat{c}_{l}$ describing that the lattice is tilted with on-site energies $-l F d$. This term can be removed by making a unitary transform with the operator $\exp \left(i F d t \sum_{l} l \hat{c}_{l}^{\dagger} \hat{c}_{l}\right)$. Then following the same process as performed in Sec. II, we obtain the resulting periodic Hamiltonian in the Bloch basis as

$$
\begin{aligned}
\hat{\mathcal{H}}_{q}^{\prime}(t)= & -\Delta \cos \phi \cos [(q+F t) d] \hat{\mathbb{1}}+\frac{\Omega}{2} \hat{\sigma}_{x} \\
& +\left\{-\frac{\delta}{2}+\Delta \sin \phi \sin [(q+F t) d]\right\} \hat{\sigma}_{z} .
\end{aligned}
$$

Introducing $\quad c_{\uparrow(\downarrow)}(q, t)=\tilde{c}_{\uparrow(\downarrow)}(q, t) \exp \left\{ \pm i \delta t / 2+i \Delta \int_{0}^{t} d t^{\prime}\right.$ $\left.\cos \left[\left(q+F t^{\prime}\right) d \pm \phi\right]\right\}$ to remove the diagonal terms in the equations of motion, we have

$$
i \frac{\partial}{\partial t}\left(\begin{array}{c}
\tilde{c}_{\uparrow}(q, t) \\
\tilde{c}_{\downarrow}(q, t)
\end{array}\right)=\frac{\Omega}{2}\left[\cos \left(\phi_{D}\right) \hat{\sigma}_{x}+\sin \left(\phi_{D}\right) \hat{\sigma}_{y}\right]\left(\begin{array}{l}
\tilde{c}_{\uparrow}(q, t) \\
\tilde{c}_{\downarrow}(q, t)
\end{array}\right),
$$

where $\phi_{D}(q, t)=\delta t+(2 \Delta \sin \phi / F d)\{\cos [(q+F t) d]-\cos$ $(q d)\}$ is the dynamical phase between the two legs. In the case of weak interleg coupling $\Omega$ (which is indeed the case we are studying), one can have

$$
\begin{aligned}
\left(\begin{array}{c}
\tilde{c}_{\uparrow}(q, t) \\
\tilde{c}_{\downarrow}(q, t)
\end{array}\right)= & \exp \left\{-i \frac{\Omega}{2} \int_{0}^{t} d t^{\prime}\left[\cos \left[\phi_{D}\left(q, t^{\prime}\right)\right] \hat{\sigma}_{x}\right.\right. \\
& \left.\left.+\sin \left[\phi_{D}\left(q, t^{\prime}\right)\right] \hat{\sigma}_{y}\right]\right\}\left(\begin{array}{c}
\tilde{c}_{\uparrow}(q, 0) \\
\tilde{c}_{\downarrow}(q, 0)
\end{array}\right),
\end{aligned}
$$

which can be recognized as Magnus expansion to the first order [32]. For atoms initially prepared in the spin- $\uparrow$ leg, the spin transition probability is given by $P_{\downarrow}=\sin ^{2}(\Omega|\chi| / 2)$ with

$$
\begin{aligned}
\chi & =\int_{0}^{t} d t^{\prime} e^{i \phi_{D}\left(q, t^{\prime}\right)} \\
& =2 \sum_{n} J_{n}(z) e^{i \delta_{n} t+i n(q d+\pi / 2)-i z \cos (q d)} \frac{\sin \left(\delta_{n} t / 2\right)}{\delta_{n}},
\end{aligned}
$$

where $z=2(\Delta / F d) \sin \phi, \delta_{n}=\delta+n F d$, and $J_{n}(z)$ is the $n$ th-order Bessel function of the first kind. Without loss of generality here we assume $q d=-\pi / 2$, which can always be achieved by shifting the starting time. Then the transition probability $P_{\downarrow}$ reads

$$
P_{\downarrow}(t)=\sin ^{2}\left[\Omega\left|\sum_{n} J_{n}(z) e^{i \delta_{n} t} \frac{\sin \left(\delta_{n} t / 2\right)}{\delta_{n}}\right|\right] .
$$




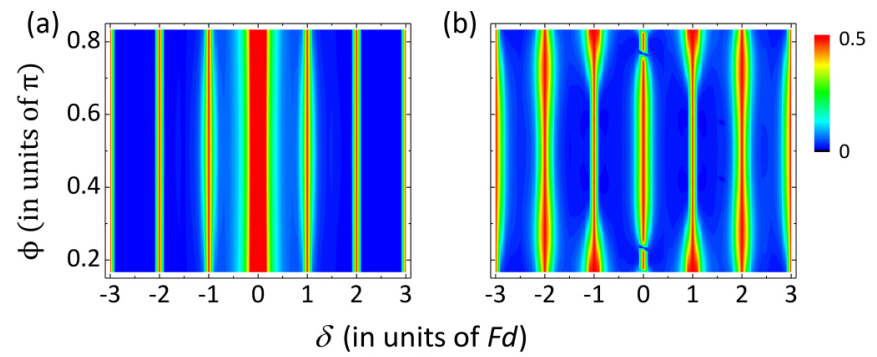

FIG. 4. Contour plot of the averaged probability $\overline{P_{\downarrow}}$ in the $(\delta, \phi)$ parameter plane with $\Omega=0.2 F d$ and (a) $\Delta=0.5 F d$ and (b) $\Delta=$ $2 F d$. The numerical simulations were performed with Eq. (13).

At some specific values of $n=m$ with $\delta_{m} \sim 0$ an interleg coupling is on resonance; then Eq. (13) becomes

$$
P_{\downarrow}(t)=\sin ^{2}\left[\Omega\left|J_{m}(z) \frac{t}{2}+\sum_{n \neq m} J_{n}(z) e^{i \delta_{n} t} \frac{\sin \left(\delta_{n} t / 2\right)}{\delta_{n}}\right|\right],
$$

which typically represents a large-period [determined by $\left.2 \pi / \Omega J_{m}(z)\right]$ oscillation with low-amplitude, high-frequency oscillations superimposed on it. Equation (14) indicates that in the long run the first term will dominate over other interleg couplings, thus the averaged probability $\overline{P_{\downarrow}}=1 / 2$. In the case where no resonance takes place, and in the meanwhile if $z \leqslant 1$, the $n=0$ term will dominate and then one has

$$
\begin{aligned}
P_{\downarrow}(t) & \approx \sin ^{2}\left[\frac{\Omega J_{0}(z)}{\delta} \sin \left(\frac{\delta t}{2}\right)\right] \\
& =\left\{2 \sum_{n=1}^{\infty} J_{2 n-1}\left(V_{0}\right) \sin \left[\left(n-\frac{1}{2}\right) \delta t\right]\right\}^{2},
\end{aligned}
$$

which can be approximately reduced to $V_{0}^{2} \sin ^{2}(\delta t / 2)$ if $\left|V_{0}\right|=\left|\Omega J_{0}(z) / \delta\right| \ll 1$. This will lead to an averaged probability of $\overline{P_{\downarrow}}=V_{0}^{2} / 2$. Combining all the discussion above, one can understand that the averaged transition probability $\overline{P_{\downarrow}}$ will vanish at large values of $\delta$ unless resonances take place at $\delta=n F d$.

We plot the averaged probability $\overline{P_{\downarrow}}$ as a function of $\delta$ and $\phi$ in Fig. 4 based on Eq. (13). Figure 4(a) represents $z<1$; Fig. 4(b), $z>1$. In both cases the resonances around $\delta=n F d$ can be clearly observed, indicating that the system can be used for force detection. The precision of force detection is related to the uncertainty of two-photon detuning $\delta$, which can be restricted, for example, by performing Pound-Drever-Hall laser frequency stabilization on the Raman beams. Figure 4(a) shows a weak dependence of $\overline{P_{\downarrow}}$ on $\phi$, however, at $z>1$ one can still expect differences while varying $\phi$, as displayed in Fig. 4(b). In this figure one can even observe that on the $\delta=0$ resonance $\overline{P_{\downarrow}}$ vanishes at some specific values of $\phi$, which correspond to the points with $J_{0}(z)=0$.

\section{CHIRAL BLOCH OSCILLATION}

In the case of a weak force $F$ and large interleg coupling $\Omega$, i.e., no interband transitions, the traditional Bloch oscillation takes place in the present system with a period $T_{B}=2 \pi / F d$ and an amplitude proportional to the bandwidth [70,71]. For Hamiltonian (2), at zero detuning $\delta=0$ the eigenstate of the
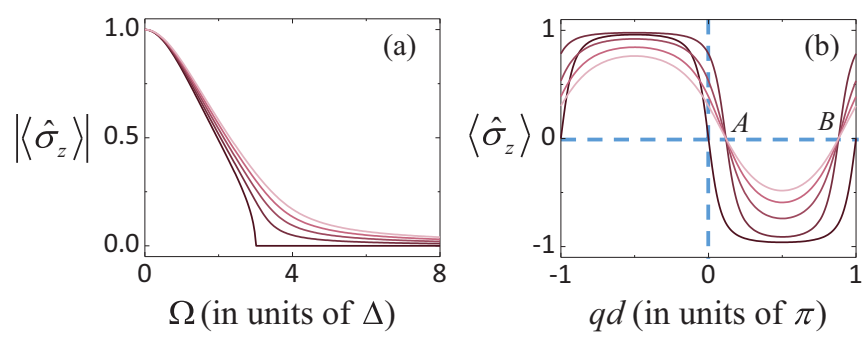

FIG. 5. (a) $\left|\left\langle\hat{\sigma}_{z}\right\rangle\right|$ of the ground state as a function of $\Omega$. From dark to light, $\delta=00.05 \Delta, 0.1 \Delta, 0.15 \Delta$, and $0.2 \Delta$, respectively. (b) $\left\langle\hat{\sigma}_{z}\right\rangle$ of the lowest band. The darkest line is for $\delta=0$ and $\Omega=0.5 \Delta$, indicating that the eigenvectors in the whole band have negative chirality. The other lines, from dark to light, are for $\delta=$ $0.63 \Delta$ and $\Omega=0.5 \Delta, \Delta, 1.5 \Delta$, and $2 \Delta$. The synthetic magnetic flux $\phi=\pi / 3$.

lowest (highest) energy band has negative (positive) chirality. The chirality can be defined as $\mathcal{C}=q\left(\left\langle\hat{c}_{q \uparrow}^{\dagger} \hat{c}_{q \uparrow}\right\rangle-\left\langle\hat{c}_{q \downarrow}^{\dagger} \hat{c}_{q \downarrow}\right\rangle\right)=$ $q\left\langle\hat{\sigma}_{z}\right\rangle$. Then in the approximation under which the atoms move adiabatically along the energy band, the Bloch oscillation will exhibit chiral characteristics [49]. For an atomic wave packet moving along the lowest band with negative chirality, the positive (negative) momentum will tend to associate with spin- $\downarrow$ (spin- $\uparrow$ ) atoms, signaling spin-momentum locking. In addition, at $\delta=0$ and a small interleg coupling $\Omega$, the lowest energy band can have two energy degenerate minima, characteristic of spin-orbit-coupled systems. Increasing $\Omega$ beyond a critical value, the two minima will merge into one minimum, signaling a quantum phase transition from the vortex phase to the Meissner phase, which was experimentally observed in [38]. This band curvature change upon the phase transition can also be captured via Bloch oscillation [49].

At any finite $\delta$, the energy band is asymmetric with respect to inversion in the Brillouin zone $(q \rightarrow-q)$, which breaks time-reversal symmetry. As a result of time-reversal symmetry breaking, Kramers degeneracy does not hold here. However, the lowest energy band can still exhibit two nondegenerate local minima, typical of SO-coupled systems such as those demonstrated in [39]. In Fig. 5(a), where $\left|\left\langle\hat{\sigma}_{z}\right\rangle\right|$ of the ground state is plotted as a function of $\Omega$, the first-order derivative discontinuity disappears at any finite $\delta$, indicating that no quantum phase transition exists. In the meanwhile, chirality is not always negative for all the eigenstates in the lowest energy band, as shown in Fig. 5(b). For the parameters considered here, at finite detuning one can have negative chirality in the quasimomentum region $[-\pi, 0]$ and $\left[q_{A}, q_{B}\right]$, where $q_{A(B)}$ are the same as defined in Sec. III, while in the rest of the first Brillouin zone the chirality becomes positive for ground eigenstates. This chirality change will be reflected in the Bloch oscillation dynamics.

We simulate the Bloch oscillation dynamics using the method of eigenstate expansion developed in [71]. Initially the atoms are assumed to be prepared in a state $|\psi\rangle=$ $\sum_{l, \sigma} \psi_{l, \sigma} \hat{c}_{l, \sigma}^{\dagger}|0\rangle$ with

$$
\psi_{l}(t=0)=\frac{1}{(2 w \sqrt{\pi})^{1 / 2}} e^{-\left(l-l_{0}\right)^{2} / 2 w^{2}+i q_{0} l d}\left(\begin{array}{l}
1 \\
1
\end{array}\right),
$$




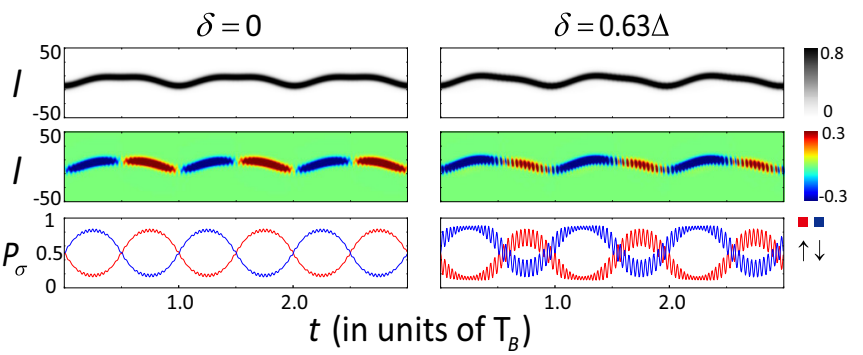

FIG. 6. Bloch oscillation dynamics of $\left|\psi_{l, \uparrow}\right|^{2}+\left|\psi_{l, \downarrow}\right|^{2}$ (top row), $\left|\psi_{l, \uparrow}\right|^{2}-\left|\psi_{l, \downarrow}\right|^{2}$ (middle row), and $\sum_{l}\left|\psi_{l, \sigma}\right|^{2}$ (bottom row). The left column corresponds to $\delta=0$, while the right column corresponds to $\delta=0.63 \Delta$. The other parameters are set as $\Omega=2 \Delta, \phi=\pi / 3$, and $F d=0.08 \Delta$. The initial state is given by Eq. (16) with $w=5$, $l_{0}=0$, and $q_{0}=0$.

representing a spin-balanced Gaussian wave packet with width $w$, center $l_{0}$, and initial quasimomentum $q_{0}$. The results are shown in Fig. 6. The left column represents $\delta=0$. The Bloch oscillation in the top row clearly reflects the twodegenerate-minima nature of the lowest band in the regime of the vortex phase. In the meanwhile the middle and bottom rows also show clear evidence of chiral Bloch oscillation and spin-momentum locking. The case of nonzero $\delta$ is shown in the right column. Despite the fact that the initial state, (16), is not exactly the eigenstate, the atoms can still adiabatically follow the lowest band and exhibit its curvature with two local minima. This process is associated with chirality change, as shown in the middle and bottom rows. Spin-momentum locking still exists, however, it becomes nonmonotonic: The spin- $\uparrow($ spin- $\downarrow)$ atoms do not simply associate with negative (positive) momentum, but now they are associated with a certain momentum range, which can be tuned by varying the detuning $\delta$.

\section{SUMMARY AND OUTLOOK}

We note that physically the Raman lasers inducing SO interaction also inevitably couple atoms to high-lying bands, which will affect the single-particle physics [72,73] such as dynamical instability [39]. This heating problem can be circumvented with an optical clock transition [34-36], which connects the long-lifetime states with a momentum transfer. One can perform a gauge transformation in the quasimomentum space and work with a quasimomentum-shifted band structure. Taking atom collisions into account will lead to nonlinear LZ tunneling [74] and deformation of interference patterns [75], which will be left for future investigation. The Stückelberg interferometer can still be implemented at a low interaction energy per site $(\ll \mathrm{kHz})$, which can be tuned by means of Feshbach resonance [76].

Besides the Stokes phase accumulated at the LZ transitions and the dynamical phase accumulated during adiabatical evolution between the LZ transitions, Stückelberg interference also depends on a noncyclic geometric phase. This noncyclic geometric phase is nonvanishing in special energy spectral configurations such as those with Dirac cones [77]. The geometric phase can also be made gauge dependent, for example, as proposed in a periodically driven two-level system [78,79]. A recent experiment has also tested theory for the noncyclic geometric phase [80]. It would be interesting to consider the geometric Stückelberg interferometer in future work and extend the discussion to the non-Hermitian case [81].

In summary, we have shown that SO-coupled cold atoms trapped in an optical lattice can be used to implement Stückelberg interference. It represents atom interferometry with synthetic gauge fields and provides new opportunities to measure the synthetic gauge field. The time-dependent and time-averaged spin probability is derived using Floquet-Bloch theory. Based on this the interference patterns are computed in the parameter space directly accessible in experiments and resonances are found. Finally, we studied chiral Bloch oscillation and found that the system can display a rich spin-momentum locking upon varying the detuning. The phenomena predicted in this work can be readily observed in current available experiments on atomic flux lattices.

\section{ACKNOWLEDGMENTS}

We thank Yongping Zhang for helpful discussions. This work was supported by the National Natural Science Foundation of China (Grants No. 12074120, No. 11374003, and No. 11774093), the National Key Research and Development Program of China (Grant No. 2016YFA0302001), and the Science and Technology Commission of Shanghai Municipality (Grant No. 20ZR1418500).
[1] For a review, see A. D. Cronin, J. Schmiedmayer, and D. E. Pritchard, Optics and interferometry with atoms and molecules, Rev. Mod. Phys. 81, 1051 (2009), and references therein.

[2] S. Dimopoulos, P. W. Graham, J. M. Hogan, and M. A. Kasevich, Testing General Relativity with Atom Interferometry, Phys. Rev. Lett. 98, 111102 (2007).

[3] S. Dimopoulos, P. W. Graham, J. M. Hogan, and M. A. Kasevich, General relativistic effects in atom interferometry, Phys. Rev. D 78, 042003 (2008).

[4] H. Müller, S.-w. Chiow, S. Herrmann, S. Chu, and K.-Y. Chung, Atom-Interferometry Tests of the Isotropy of Post-Newtonian Gravity, Phys. Rev. Lett. 100, 031101 (2008).
[5] T. van Zoest et al., Bose-Einstein condensation in microgravity, Science 328, 1540 (2010).

[6] Y. Margalit, Z. Zhou, S. Machluf, D. Rohrlich, Y. Japha, and R. Folman, A self-interfering clock as a "which path" witness, Science 349, 1205 (2015).

[7] B. M. Anderson, J. M. Taylor, and V. M. Galitski, Interferometry with synthetic gauge fields, Phys. Rev. A 83, 031602(R) (2011).

[8] Y.-X. Du, H. Yan, D.-W. Zhang, C.-J. Shan, and S.-L. Zhu, Proposal for a rotation-sensing interferometer with spin-orbit-coupled atoms, Phys. Rev. A 85, 043619 (2012). 
[9] A. Jacob, P. Öhberg, G. Juzeliūnas, and L. Santos, Cold atom dynamics in non-Abelian gauge fields, Appl. Phys. B 89, 439 (2007).

[10] K. Osterloh, M. Baig, L. Santos, P. Zoller, and M. Lewenstein, Cold Atoms in Non-Abelian Gauge Potentials: From the Hofstadter "Moth" to Lattice Gauge Theory, Phys. Rev. Lett. 95, 010403 (2005).

[11] Y.-J. Lin, K. Jiménez-García, and I. B. Spielman, Spin-orbitcoupled Bose-Einstein condensates, Nature (London) 471, 83 (2011).

[12] A. Celi, P. Massignan, J. Ruseckas, N. Goldman, I. B. Spielman, G. Juzeliūnas, and M. Lewenstein, Synthetic Gauge Fields in Synthetic Dimensions, Phys. Rev. Lett. 112, 043001 (2014).

[13] J. Dalibard, F. Gerbier, G. Juzeliūnas, and P. Öhberg, Colloquium: Artificial gauge potentials for neutral atoms, Rev. Mod. Phys. 83, 1523 (2011).

[14] Y. Yang, C. Peng, D. Zhu, H. Buljan, J. D. Joannopoulos, B. Zhen, and M. Soljačić, Synthesis and observation of non-Abelian gauge fields in real space, Science 365, 1021 (2019).

[15] S. Sugawa, F. Salces-Carcoba, A. R. Perry, Y. Yue, and I. B. Spielman, Second Chern number of a quantum-simulated nonAbelian Yang monopole, Science 360, 1429 (2018).

[16] S. Sugawa, F. Salces-Carcoba, Y. Yue, A. Putra, and I. B. Spielman, Observation and characterization of a nonAbelian gauge field's Wilczek-Zee phase by the Wilson loop, arXiv:1910.13991.

[17] L. D. Landau, On the theory of transfer of energy at collisions II, Phys. Z. Sowjetunion 2, 46 (1932); C. Zener, Non-adiabatic crossing of energy levels, Proc. R. Soc. Ser. A 137, 696 (1932).

[18] E. C. G. Stückelberg, Theory of inelastic collisions between atoms, Helv. Phys. Acta 5, 369 (1932).

[19] S. N. Shevchenko, S. Ashhab, and F. Nori, Landau-ZenerStückelberg interferometry, Phys. Rep. 492, 1 (2010).

[20] H. Nakamura, Nonadiabatic Transition: Concepts, Basic Theories and Applications, 2nd ed. (World Scientific, Singapore, 2012).

[21] F. Haake, Quantum Signatures of Chaos (Springer-Verlag, Berlin, 1991).

[22] M. Mark, T. Kraemer, P. Waldburger, J. Herbig, C. Chin, H.C. Nägerl, and R. Grimm, "Stückelberg Interferometry" with Ultracold Molecules, Phys. Rev. Lett. 99, 113201 (2007).

[23] S. Kling, T. Salger, C. Grossert, and M. Weitz, Atomic BlochZener Oscillations and Stückelberg Interferometry in Optical Lattices, Phys. Rev. Lett. 105, 215301 (2010).

[24] A. Zenesini, D. Ciampini, O. Morsch, and E. Arimondo, Observation of Stückelberg oscillations in accelerated optical lattices, Phys. Rev. A 82, 065601 (2010).

[25] P. Huang, J. Zhou, F. Fang, X. Kong, X. Xu, C. Ju, and J. $\mathrm{Du}$, Landau-Zener-Stückelberg Interferometry of a Single Electronic Spin in a Noisy Environment, Phys. Rev. X 1, 011003 (2011).

[26] M. Kervinen, J. E. Ramírez-Muñoz, A. Välimaa, and M. A. Sillanpää, Landau-Zener-Stückelberg Interference in a Multimode Electromechanical System in the Quantum Regime, Phys. Rev. Lett. 123, 240401 (2019).

[27] D. Witthaut, F. Trimborn, V. Kegel, and H. J. Korsch, Quantum dynamics of Bose-Einstein condensates in tilted and driven bichromatic optical lattices, Phys. Rev. A 83, 013609 (2011).
[28] X. Cai, S. Chen, and Y. Wang, Quantum dynamics of hard-core bosons in tilted bichromatic optical lattices, Phys. Rev. A 84, 033605 (2011).

[29] T. Yamakoshi and S. Watanabe, Fast and selective interband transfer of ultracold atoms in bichromatic lattices permitting Dirac points, Phys. Rev. A 99, 013621 (2019).

[30] Z. Wang, B. Yang, D. Hu, X. Chen, H. Xiong, B. Wu, and $\mathrm{X}$. Zhou, Observation of quantum dynamical oscillations of ultracold atoms in the $\mathbf{F}$ and $\mathbf{D}$ bands of an optical lattice, Phys. Rev. A 94, 033624 (2016).

[31] N. Lörch, F. V. Pepe, H. Lignier, D. Ciampini, R. Mannella, O. Morsch, E. Arimondo, P. Facchi, G. Florio, S. Pascazio, and $\mathrm{S}$. Wimberger, Wave-function-renormalization effects in resonantly enhanced tunneling, Phys. Rev. A 85, 053602 (2012).

[32] P. Plötz and S. Wimberger, Stückelberg-interferometry with ultra-cold atoms, Eur. Phys. J. D 65, 199 (2011).

[33] A. J. Olson, D. B. Blasing, C. Qu, C.-H. Li, R. J. Niffenegger, C. Zhang, and Y. P. Chen, Stueckelberg interferometry using periodically driven spin-orbit-coupled Bose-Einstein condensates, Phys. Rev. A 95, 043623 (2017).

[34] L. F. Livi, G. Cappellini, M. Diem, L. Franchi, C. Clivati, M. Frittelli, F. Levi, D. Calonico, J. Catani, M. Inguscio, and L. Fallani, Synthetic Dimensions and Spin-Orbit Coupling with an Optical Clock Transition, Phys. Rev. Lett. 117, 220401 (2016).

[35] M. L. Wall, A. P. Koller, S. Li, X. Zhang, N. R. Cooper, J. Ye, and A. M. Rey, Synthetic Spin-Orbit Coupling in an Optical Lattice Clock, Phys. Rev. Lett. 116, 035301 (2016).

[36] S. Kolkowitz, S. L. Bromley, T. Bothwell, M. L. Wall, G. E. Marti, A. P. Koller, X. Zhang, A. M. Rey and J. Ye, Spin-orbitcoupled fermions in an optical lattice clock, Nature (London) 542, 66 (2017).

[37] J. Li, W. Huang, B. Shteynas, S. Burchesky, F. C. Top, E. Su, J. Lee, A. O. Jamison, and W. Ketterle, Spin-Orbit Coupling and Spin Textures in Optical Superlattices, Phys. Rev. Lett. 117, 185301 (2016).

[38] M. Atala, M. Aidelsburger, M. Lohse, J. T. Barreiro, B. Paredes, and I. Bloch, Observation of the Meissner effect with ultracold atoms in bosonic ladders, Nat. Phys. 10, 588 (2014).

[39] C. Hamner, Y. Zhang, M. A. Khamehchi, M. J. Davis, and P. Engels, Spin-Orbit-Coupled Bose-Einstein Condensates in a One-Dimensional Optical Lattice, Phys. Rev. Lett. 114, 070401 (2015).

[40] Z. Wu, L. Zhang, W. Sun, X.-T. Xu, B.-Z. Wang, S.-C. Ji, Y. Deng, S. Chen, X.-J. Liu, and J.-W. Pan, Realization of twodimensional spin-orbit coupling for Bose-Einstein condensates, Science 354, 83 (2016).

[41] F. A. An, E. J. Meier, and B. Gadway, Direct observation of chiral currents and magnetic reflection in atomic flux lattices, Sci. Adv. 3, e1602685 (2017).

[42] D. Hügel and B. Paredes, Chiral ladders and the edges of quantum Hall insulators, Phys. Rev. A 89, 023619 (2014).

[43] A. Dutt, Q. Lin, L. Yuan, M. Minkov, M. Xiao, and S. Fan, A single photonic cavity with two independent physical synthetic dimensions, Science 367, 59 (2020).

[44] R. Takahashi and N. Sugimoto, Semiclassical lattice effects on interband tunneling of a two-state system, J. Phys. Soc. Jpn. 87, 104701 (2018).

[45] R. Takahashi and N. Sugimoto, Current control of systems with a Peierls distortion by magnetic field, Phys. Rev. B 98, 155111 (2018). 
[46] S. S. Natu, Bosons with long-range interactions on two-leg ladders in artificial magnetic fields, Phys. Rev. A 92, 053623 (2015).

[47] S. Greschner, M. Piraud, F. Heidrich-Meisner, I. P. McCulloch, U. Schollwöck, and T. Vekua, Symmetry-broken states in a system of interacting bosons on a two-leg ladder with a uniform Abelian gauge field, Phys. Rev. A 94, 063628 (2016).

[48] S. K. Ghosh, S. Greschner, U. K. Yadav, T. Mishra, M. Rizzi, and V. B. Shenoy, Unconventional phases of attractive Fermi gases in synthetic Hall ribbons, Phys. Rev. A 95, 063612 (2017).

[49] Y. Zheng, S. Feng, and S.-J. Yang, Chiral Bloch oscillation and nontrivial topology in a ladder lattice with magnetic flux, Phys. Rev. A 96, 063613 (2017).

[50] A. Petrescu, M. Piraud, G. Roux, I. P. McCulloch, and K. L. Hur, Precursor of the Laughlin state of hard-core bosons on a two-leg ladder, Phys. Rev. B 96, 014524 (2017).

[51] S. Barbarino, M. Dalmonte, R. Fazio, and G. E. Santoro, Topological phases in frustrated synthetic ladders with an odd number of legs, Phys. Rev. A 97, 013634 (2018).

[52] R. Citro, S. De Palo, M. Di Dio, and E. Orignac, Quantum phase transitions of a two-leg bosonic ladder in an artificial gauge field, Phys. Rev. B 97, 174523 (2018).

[53] T. Haug, L. Amico, R. Dumke and L.-C. Kwek, Mesoscopic vortex-Meissner currents in ring ladders, Quantum Sci. Technol. 3, 035006 (2018).

[54] J. H. Han, J. H. Kang, and Y. Shin, Band Gap Closing in a Synthetic Hall Tube of Neutral Fermions, Phys. Rev. Lett. 122, 065303 (2019).

[55] M. Mancini, G. Pagano, G. Cappellini, L. Livi, M. Rider, J. Catani, C. Sias, P. Zoller, M. Inguscio, M. Dalmonte, and L. Fallani, Observation of chiral edge states with neutral fermions in synthetic Hall ribbons, Science 349, 1510 (2015).

[56] A. Haller, M. Rizzi, and M. Burrello, The resonant state at filling factor $v=1 / 2$ in chiral fermionic ladders, New J. Phys. 20, 053007 (2018).

[57] J. Jünemann, A. Piga, S.-J. Ran, M. Lewenstein, M. Rizzi, and A. Bermudez, Exploring Interacting Topological Insulators with Ultracold Atoms: The Synthetic Creutz-Hubbard Model, Phys. Rev. X 7, 031057 (2017).

[58] M. C. Strinati, E. Cornfeld, D. Rossini, S. Barbarino, M. Dalmonte, R. Fazio, E. Sela, and L. Mazza, Laughlin-Like States in Bosonic and Fermionic Atomic Synthetic Ladders, Phys. Rev. X 7, 021033 (2017).

[59] B. K. Stuhl, H.-I. Lu, L. M. Aycock, D. Genkina, and I. B. Spielman, Topological phases of fermionic ladders with periodic magnetic fields, Science 349, 1514 (2015).

[60] M. Eric Tai, A. Lukin, M. Rispoli, R. Schittko, T. Menke, D. Borgnia, P. M. Preiss, F. Grusdt, A. M. Kaufman, and M. Greiner, Microscopy of the interacting Harper-Hofstadter model in the few-body limit, Nature 546, 519 (2017).

[61] W. Tschischik, R. Moessner, and M. Haque, Bose-Hubbard ladder subject to effective magnetic field: Quench dynamics in a harmonic trap, Phys. Rev. A 92, 023845 (2015).

[62] D. Genkina, L. M. Aycock, H.-I Lu, M. Lu, A. M. Pineiro, and I. B. Spielman, Imaging topology of Hofstadter ribbons, New J. Phys. 21, 053021 (2019).

[63] $\Delta$ is estimated with an optical lattice potential $U_{0}=-1.4 E_{l}$, where $E_{l}=\hbar^{2} k_{l}^{2} / 2 m$ with $k_{l} \approx 1540 \mathrm{~nm}$ [39].

[64] M. B. Dahan, E. Peik, J. Reichel, Y. Castin, and C. Salomon, Bloch Oscillations of Atoms in an Optical Potential, Phys. Rev.
Lett. 76, 4508 (1996); E. Peik, M. B. Dahan, I. Bouchoule, Y. Castin, and C. Salomon, Bloch oscillations of atoms, adiabatic rapid passage, and monokinetic atomic beams, Phys. Rev. A 55, 2989 (1997).

[65] B. M. Garraway and N. V. Vitanov, Population dynamics and phase effects in periodic level crossings, Phys. Rev. A 55, 4418 (1997).

[66] B. Damski and W. H. Zurek, Adiabatic-impulse approximation for avoided level crossings: From phase-transition dynamics to Landau-Zener evolutions and back again, Phys. Rev. A 73, 063405 (2006).

[67] J. Rotvig, A.-P. Jauho, and H. Smith, Bloch Oscillations, Zener Tunneling, and Wannier-Stark Ladders in the Time Domain, Phys. Rev. Lett. 74, 1831 (1995); Theory of coherent time-dependent transport in one-dimensional multiband semiconductor superlattices, Phys. Rev. B 54, 17691 (1996).

[68] X.-G. Zhao, G. A. Georgakis, and Q. Niu, Rabi oscillations between Bloch bands, Phys. Rev. B 54, R5235 (1996).

[69] A. R. Kolovsky and A. Buchleitner, Floquet-Bloch operator for the Bose-Hubbard model with static field, Phys. Rev. E 68 , 056213 (2003).

[70] Y. V. Kartashov, V. V. Konotop, D. A. Zezyulin, and L. Torner, Bloch Oscillations in Optical and Zeeman Lattices in the Presence of Spin-Orbit Coupling, Phys. Rev. Lett. 117, 215301 (2016).

[71] W. Ji, K. Zhang, W. Zhang, and L. Zhou, Bloch oscillations of spin-orbit-coupled cold atoms in an optical lattice and spincurrent generation, Phys. Rev. A 99, 023604 (2019).

[72] L. Zhou and X. Cui, Spin-orbit coupled ultracold gases in optical lattices: High-band physics and insufficiency of tightbinding models, Phys. Rev. B 92, 140502(R) (2015).

[73] J.-S. Pan, W. Zhang, W. Yi, and G.-C. Guo, Bose-Einstein condensate in an optical lattice with Raman-assisted twodimensional spin-orbit coupling, Phys. Rev. A 94, 043619 (2016).

[74] Y. Zhang, Z. Gui, and Y. Chen, Nonlinear dynamics of a spin-orbit-coupled Bose-Einstein condensate, Phys. Rev. A 99, 023616 (2019).

[75] S.-C. Li, L.-B. Fu, and J. Liu, Nonlinear Landau-ZenerStückelberg-Majorana interferometry, Phys. Rev. A 98, 013601 (2018).

[76] A. Marte, T. Volz, J. Schuster, S. Dürr, G. Rempe, E. G. M. van Kempen, and B. J. Verhaar, Feshbach Resonances in Rubidium 87: Precision Measurement and Analysis, Phys. Rev. Lett. 89, 283202 (2002)

[77] L.-K. Lim, J.-N. Fuchs, and G. Montambaux, Mass and Chirality Inversion of a Dirac Cone Pair in Stückelberg Interferometry, Phys. Rev. Lett. 112, 155302 (2014); Geometric phase in Stückelberg interferometry, Phys. Rev. A 91, 042119 (2015).

[78] S. Gasparinetti, P. Solinas, and J. P. Pekola, Geometric LandauZener Interferometry, Phys. Rev. Lett. 107, 207002 (2011).

[79] J. Zhang, J. Zhang, X. Zhang, and K. Kim, Realization of geometric Landau-Zener-Stückelberg interferometry, Phys. Rev. A 89, 013608 (2014).

[80] Z. Zhou, Y. Margalit, S. Moukouri, Y. Meir, and R. Folman, An experimental test of the geodesic rule proposition for the noncyclic geometric phase, Sci. Adv. 6, eaay8345 (2020).

[81] X. Shen, F. Wang, Z. Li, and Z. Wu, Landau-Zener-Stükelberg interferometry in PT-symmetric non-Hermitian models, Phys. Rev. A 100, 062514 (2019). 